A N N A L E S Annales de Bretagne et des Pays de l'Ouest

Anjou. Maine. Poitou-Charente. Touraine

108-4 | 2001

La Chambre des comptes de Bretagne

\title{
Chahut à la Chambre ou le premier président contesté septembre 1642
}

\section{Vincent Gallais}

\section{(2) OpenEdition}

Journals

\section{Édition électronique}

URL : http://journals.openedition.org/abpo/1684

DOI : $10.4000 /$ abpo.1684

ISBN : $978-2-7535-1484-3$

ISSN : 2108-6443

Éditeur

Presses universitaires de Rennes

Édition imprimée

Date de publication : 20 décembre 2001

Pagination : 159-168

ISBN : 978-2-86847-674-6

ISSN : 0399-0826

\section{Référence électronique}

Vincent Gallais, "Chahut à la Chambre ou le premier président contesté septembre 1642 », Annales de Bretagne et des Pays de l'Ouest [En ligne], 108-4 | 2001, mis en ligne le 20 décembre 2003, consulté le 19 avril 2019. URL : http://journals.openedition.org/abpo/1684 ; DOI : 10.4000/abpo.1684 


\title{
Chahut à la Chambre ou le premier président contesté septembre 1642
}

\author{
Vincent GALLAIS \\ Doctorant, CRHMA - Université de Nantes
}

Dans la société d'ordres, pétrie de privilèges et de convenances, la question des préséances prend une place primordiale tant l'énergie déployée par ses membres pour la défense des prérogatives du rang, tacites ou officielles, est forte. Le théâtre judiciaire n'échappe pas à cette réalité : prévenir les usurpations et veiller au respect de la hiérarchie des grades constituent des gestes presque naturels pour les garants de l'ordre royal ${ }^{1}$. L'exemple du désordre qui surprend, César-Auffray Blanchard, premier président de la Chambre des comptes de Bretagne, en septembre 1642, nous livre une illustration du type de querelle engendrée par l'obsédante distinction du paraître. En dépit du prestige de sa fonction, quelques agitateurs s'opposent à lui et perturbent les cérémonies d'ouverture du second semestre; l'examen de cette affaire nous amène à rappeler le rôle de chacun des acteurs, à préciser les faits et enfin, à exposer les suites et non-dits de l'intrigue ${ }^{2}$.

\section{Du cérémonial de rentrée}

L'inauguration des séances semestrielles - en mars et septembre - qui annonce la reprise des travaux judiciaires s'ordonne autour d'un cérémonial où se succèdent plusieurs étapes qui forment autant de célébrations de l'union et force du corps : ouverture des portes de la Chambre, procession et messe au couvent des cordeliers ${ }^{3}$, audience solennelle rythmée

1. Robert Muchembled, Politique et politesse en France du XVI au XXe siècle, Paris, 1998, p. $80-83$.

2. Arch. dép. de Loire-Atlantique, $103 \mathrm{~J}$ 17, procez verbal du s[ieu]r Blanchard premier president des comptes a Nantes du trouble qui luy a esté fait par le s[ieu]r du Pont et autres presidents des[dit]s comptes

3. Les relations de la cour avec cette congrégation sont étroites depuis l'origine même de son installation au bord de l'Erdre. En 1614, les religieux exprimèrent leur intention, non suivie d'effets, de quitter le quartier pour s'installer " aux forbourgs " et les officiers 
par la lecture des ordonnances et le discours du premier président suivis du dîner de retrouvailles des officiers.

Les personnalités impliquées dans l'affaire évoluent dans le même espace social et professionnel, celui de la haute judicature bretonne. Après un prompt apprentissage de la magistrature comme président au présidial de Nantes, César-Auffray Blanchard (1612-1671) suit la voie paternelle qui le mène à la Chambre des comptes. Malgré la délivrance des lettres de provision de l'office de premier président, en décembre 1635, il doit néanmoins attendre le 27 juillet 1641 pour être admis au prestigieux fauteuil. En effet, en 1637, son père, Jean Blanchard, déjà en charge, obtient du souverain le privilège d'exercice de la haute fonction pendant cinq années supplémentaires ${ }^{4}$. Concernant le principal contradicteur, Louis du Pont ${ }^{5}$, il est membre de la Chambre depuis 1623 et occupe la seconde place dans la hiérarchie, où il est en charge du semestre de septembre, et appartient à la parentèle de César-Auffray Blanchard. Son fils, Guy du Pont, conseiller au Parlement de Bretagne, a épousé en mai 1640 Françoise Becdelièvre, nièce du premier président.

En 1642, le jeune premier président doit subir l'affront de la remise en cause de sa prééminence par une minorité d'officiers conduite par ce président. Profitant de l'inexpérience du magistrat, et de l'absence de son autoritaire aîné, les juges contestataires tentent d'empêcher le dirigeant en droit de la compagnie de pratiquer personnellement, et dans les règles, l'inauguration de la session de la Chambre.

La description des fonctions respectives des perturbateurs permet de mieux comprendre les enjeux de la querelle ${ }^{6}$. Les édits royaux confèrent de larges pouvoirs au premier président, en particulier celui de siéger en permanence au grand bureau où l'on traite les affaires les plus importantes. Il est prioritaire dans la connaissance des textes législatifs expédiés

obtinrent alors des lettres patentes du roi (21 août 1614) les autorisant à " faire bastir une chapelle affin dy entendre la messe et assister au service divin pour ne plus aller aux audiences par les rues publicques jusques a leglize de saint François assés esloignée " (Arch. dép. de Loire-Atlantique, B 240, rappelé dans un arrêt du 18 août 1634). En 1650, César-Auffray Blanchard ordonne que l'on verse 15 livres aux chantres de la cathédrale " qui ont chanté la musique en leglize des reverendz pères cordelliers " à l'ouverture de la séance présente (Arch. dép. de Loire-Atlantique, B 290, arrêt du 24 mars). De même, en 1652, on accorde 87 livres aux cordeliers pour la célébration de la " sainte messe ", dite pour la séance en cours, et 12 livres, 10 sols en paiement de la fourniture de luminaires, tapis et autres coussins (Arch. dép. de Loire-Atlantique, B 294, arrêt du 20 février).

4. Arch. dép. de Loire-Atlantique, 103 J 4 et 103 J 17, Jacques Charette (1637-1677) lui succède le 18 mai 1673.

5. Louis du Pont prend la place de Trajan de La Coussaye le 13 novembre 1623. Le 9 septembre 1644, il résigne son office en faveur de Jacques Huteau ( $† 1670)$. Les renseignements relatifs à la carrière des officiers sont extraits de Gaétan d'AVIAU de TERNAY, Dictionnaire des magistrats de la Chambre des Comptes de Bretagne, 1995, un volume dactylographié.

6. Henri de Fourmont, Histoire de la Chambre des Comptes de Bretagne, Paris, 1854, p. 389-391. 
par le gouvernement monarchique et qui lui sont remis par le procureur général du roi. La diffusion des commandements royaux ou des nouvelles touchant la vie politique nationale et internationale, lui incombe également. En tant que chef du corps des magistrats comptables, il reçoit le serment des nouveaux titulaires d'office. Aucun document ni aucun fait ne lui échappe. D'ailleurs, il joue un rôle dans la distribution des tâches quotidiennes, nomme aux commissions établies par la Chambre et dirige celles instituées au Conseil du roi. Le plus haut magistrat nantais représente la cour souveraine non seulement dans les grandes occasions et les fêtes religieuses locales mais aussi dans les délégations envoyées en Cour. À chaque ouverture des sessions semestrielles, il préside l'ensemble des cérémonies tant juridiques que spirituelles.

Quant aux présidents qui officient semestriellement au second bureau, ils fixent les modalités pratiques d'exécution des ordres et orientations de leur supérieur hiérarchique et s'assurent de la bonne tenue des comptes et de la qualité et répartition du travail des auditeurs et maîtres. En outre, il leur est permis de siéger toute l'année mais avec des prérogatives extrêmement limitées hors l'espace de leur semestre. En l'absence du premier président, le plus ancien d'entre eux peut remplir ses fonctions; la mise en pratique de cette ultime faculté est à l'origine du conflit qui éclate à l'audience de rentrée de 1642.

\section{" Trouble et beau bruit "}

Au cœur de l'affrontement réside l'enjeu de la présidence de la séance inaugurale du semestre, dit de septembre, juridiquement et réglementairement dévolue au premier président mais qui, en cas d'éloignement ou d'incapacité de ce dernier, peut être suppléé par le plus ancien de ses subordonnés. Les faits débutent le lundi premier septembre 1642 à l'hôtel Blanchard où le haut magistrat reçoit, dès 7 heures du matin, la visite de Philippe Varice ${ }^{7}$, doyen des maîtres des comptes du semestre de septembre :

" Lequel nous auroit dit estre venu expres pour scavoir de nous sy nostre intention estoit dentrer ce jour en ladite chambre pour en faire louverture et nous convier a nous en abstenir dautant quil prevoict que nous y serions troublés."

L'orgueil piqué au vif, l'officier se défend par ces mots lancés à l'encontre du matinal impertinent :

" Nous trouvant en la ville le jour destiné a ladite ouverture, nous estimions quil estoit de la dignité de la compagnie et de nostre charge dentrer en ladite chambre pour y presider et quil y auroit de la messeance de nous en abstenir [...]."

7. Philippe Varice (1585-1646) fait son entrée à la Chambre le 14 juin 1614 à la place de René Le Clerc († 1639). À son décès, Jean Martin († 1672) lui succède. Il a épousé en 1608 Françoise, sœur du président Louis du Pont. 
Puis, il évoque ce qui devient aussitôt l'objet, indispensable et incontournable, sur lequel va se cristalliser l'affrontement :

"Nous y estions obligé par une autre raison qui est que nous avions esté chargée par ladite chambre dune des clefs qui ferment les bureaux a la fermeture derniere pour la rep[rése]nter a louverture du semestre qui commence ce jour $[\ldots]$. »

En retour, son visiteur le menace :

"Vous estes doncq resolu dy entrer, vous allés bien voir du trouble et beau bruit. "

César-Auffray Blanchard, clairvoyant et bien informé, ne tarde pas à apprendre le nom de l'instigateur de cette cabale protocolaire. Ainsi, il fustige le comportement de Louis du Pont et de ses complices - le maître Varice, son beau-frère et " inthime amy lié a ses interests et ayant pouvoir sur son esprit ", Christophe Juchault ${ }^{8}$ et René Le Ferron ${ }^{9}$, respectivement troisième et quatrième présidents de ce semestre - qu'il accuse d'avoir " fait les jours precedans diverses brigues pour preparer ledit trouble ".

Après avoir refusé de se plier aux injonctions de Philippe Varice et par conséquent de lui remettre sa clé, le haut magistrat s'achemine rapidement au palais des comptes où le rejoignent ses opposants vers $7 \mathrm{~h} 30$. Cependant, en cet instant, le magistrat ne prend pas encore trop au sérieux le message qui vient de lui être adressé :

" Nous ne pouvions nous persuader qui leur eust entré en lesprit de faire un attentat sy manifeste et sy public a lauctorité de sa majesté et arrests de son conseil et nous persuadant plustot que le propos que nous avoit tenu ledit sieur Varice estoit un artiffice pour nous empescher et divertir dentrer. Joint lobligation que nous avions de nous y trouver pour la reputation de la clef dont nous avions esté chargé, nous avons continué nostre dessein dentrer. "

Ensuite, les événements s'enchaînent. Aussitôt après son arrivée, le président Juchault confisque la clé insérée par le greffier, qui en a la garde, dans la serrure de la porte " qui respond sur le grand escalier " et la met " dans sa pochette a dessein comme il est apparant dempescher que les officiers de ladite chambre peussent sortir pour aller aux cordeliers pour la celebration de la messe du sainct esprit a laquelle ils ont de coustume daller et assister en corps aux ouvertures des seanses avant la lecture des ordonnances ". Déterminé, le premier président décide alors d'entraîner la compagnie vers la haute porte qui condamne l'entrée des bureaux où l'attend Pierre Ernaud ${ }^{10}$, " greffier du semestre ", qui en conserve l'une des

8. Christophe Juchault ( $\uparrow 1661$ ) obtient l'office de président sur résignation de Gabriel Turmelin; il est reçu le 24 février 1635 et quitte ses fonctions en 1643 au profit de Roland Morin († 1654).

9. René Ferron, ou Le Ferron, (1594-1651) entre en charge le 24 novembre 1628 après le départ de Victor Binet. À sa mort, son successeur est René de Pontual (1607-1698). En 1630, il s'est marié en secondes noces avec la sœur du président Christophe Juchault.

10. Pierre Ernaud ( $† 1650)$ accède en 1650 au poste de greffier en chef sur résignation de Jean Le Haste (1594-1654). En 1650, Guy Guyton lui succède. 
trois clés. Entre temps, le président du Pont, profitant du retard d'une partie des maîtres, peut-être provoqué par leur doyen Varice, " descendit en la gallerie sur laquelle respond ladite porte " afin de s'emparer, " par force " de l'une des clés en mains dudit greffier. Parvenu enfin jusqu'audit lieu, le premier président ordonne l'ouverture mais la clé que lui remet Hervé Bertaud $^{11}$, premier huissier de la chambre, et la sienne, s'avèrent bien inutiles après le passage du second président. Le magistrat perd patience et condamne l'usage d'" une telle violence de la part dun officier de sa qualité et en la maison du Roy, lieu sacré" ".

La tension augmente encore car attisée par la remarque du président Juchault qui clame dans la cohue, à destination de son supérieur et au sujet de l'ouverture convoitée : "Ce nestoit pas a vous a la faire. " César-Auffray Blanchard, bafoué dans ses prérogatives, s'emporte alors contre le greffier dépossédé de sa clé en accusant :

" Il y avoit plus daparence questant creature dudit s[ieu]r du Pont faisant ses affaires et escrivant continuellement soubz luy comme son domestiq et selon le bruit commun son redevable, il sestoit entendu avecq ledit $s[i e u] r$ du Pont pour faire ce trouble."

Suivent des échanges et " civilités " entre le premier et le second président; chacun revendique le droit de diriger la première séance du semestre, réclame l'allégeance de l'autre. Finalement, l'enlisement de la situation ne fait qu'accroître, au grand dam du chef de la compagnie, le " retardement de louverture et du service du Roy ».

Par ailleurs, le juge prend à témoin les officiers, leur demande de " se souvenir " et rappelle sans cesse que la jurisprudence constituée par les arrêts du Conseil cautionne son intransigeance. De plus, il fait rapporter ses " protestations " par Robin, commis au greffe. Puis, il tente de régler le conflit en conviant les présidents à se rendre au bureau et invite Louis du Pont à s'expliquer mais ses " prières " demeurent sans effets. En désespoir de cause, il proteste vigoureusement auprès des gens du roi - avocat et procureur général - et sollicite leur appui afin qu'ils convainquent les présidents et leurs partisans d'abréger la " continuation dudit trouble ». Malgré les menaces répétées des uns et des autres, les officiers rebelles campent sur leur position et confisquent la clé. À partir de ce moment, Philippe Varice demeure l'interlocuteur privilégié du premier président avec lequel il confère de longues heures, dans la salle de réunion du bureau, sans céder sur les revendications des présidents par lesquels il est mandaté :

"Ledit sieur Dupont ne voulant suivre aucune chose de lordre ancien et acoustumé au lieu de nous mettre entre mains le clef quil avoit la bailla audit sieur Varice, son beau-frère, qui nous donna subject de nous adresser a luy et de luy rep[résen]ter lauctorité des arrests du conseil du roy et de la chambre, lobeissance qui y devoit et lexemple quil devoit montrer aux autres offi-

11. Hervé Bertaud (1601-1648) entre en fonction le 28 mai 1625 sur le départ de Simon Picard († 1579) avant de céder sa charge en 1649 à François Rouillé († 1652). 
ciers estant lancien et le doien. Et sur son refus desclarasmes que nous le prenions a partie et privé [... Mais il persista neanmoins en son refus disant quil feroit ce que la chambre ordonnoit voulant par la nous obliger a nous retirer pour mettre ne deliber[ati]on les choses tant de foyes jugées."

Pendant ce temps, Olivier Cassard ${ }^{12}$, " garde des livres de ladite chambre ", émissaire du premier président, tente une nouvelle médiation qui reste sans issue tandis que Guillaume Macé, greffier du semestre de mars, est mandé pour renforcer le dispositif de défense du magistrat contesté. Vers 15 heures, les parties se lassent. Le premier juge des comptes déplore que ses collègues aient été retenus par des contestations dont l'objectif était de " faire couler le temps de la messe et de la lecture des ordonnances et empescher que les ceremonies acoustumées aux ouvertures eussent esté pratiquées ". Une heure plus tard, les opposants provoquent de nouveau sa colère en exprimant leur intention d'accéder aux autres salles par l'intervention d'un serrurier. Cette fois, les autres magistrats, jusque-là passifs, semblent marquer leur désapprobation en quittant la Chambre; de fait, le projet est abandonné. Pour finir, après d'ultimes appels à la sagesse, le premier président, parmi les "derniers avecq les gens du roy ", se retire à son domicile en espérant " que la nuict rallentiroit le cours de telles violances".

Le jour suivant l'incident, la victime adresse au monarque un mémoire, long et circonstancié, rédigé avec une plume dûment acérée, trempée dans une encre mêlée d'amertume et de ressentiment, pour dénoncer « un attentat »; laissons le nous exposer l'épilogue de cette pénible affaire :

"Le lendemain mardy deuxiesme dudit moys de septembre nous estans entrés en ladite chambre en esperance dy trouver lesdits s[ieu]rs presidents plus disposés a lobeissance et a souffrir lexecution des volontés du Roy et des arrests de son conseil, nous les trouvasmes en la mesme opiniatreté et eux et ledit $s[i e u] r$ Varice en resolution de faire ouvrir la porte desdits bureaux par un serrurier. Ce qui nous a fait juger plus a propos de relascher quelque chose des droits et de lautorité de nostre charge que de souffrir une action de sy grand esclat et dune sy perilleuse consequence, c'est pourquoy nous avons mis les deux clefs que nous avions entre les mains dudit Ernaud greffier et luy avons enjoint de faire raporter louverture quil feist en nostre $\mathrm{p}$ [rése]nce, ledit $\mathrm{s}[\mathrm{ieu}] \mathrm{r}$ Varice luy ayant apres nous delivré celle quil avoit [...]."

Les rites qui accompagnent la rentrée des gens des comptes n'ont pas été respectés; le premier président en fut grandement affecté. En particulier, pour sa première ouverture, César-Auffray Blanchard a donc dû renoncer à prononcer la traditionnelle harangue qui occupe pourtant une place fondamentale tant dans le rituel des cours souveraines que dans la conception éthico-professionnelle de l'orateur judiciaire ${ }^{13}$.

12. Olivier Cassard est reçu comme " garde des chartes de Bretagne " le 12 avril 1638 en remplacement de Guillaume Macé et résigne en faveur de Antoine Guyho († 1620) en 1644.

13. Voir Pierre ZoBerman, Les Cérémonies de la parole. L'éloquence d'apparat en France dans le dernier quart du XVII siècle, Paris, 1999, p. 319-532; César-Auffray Blanchard prononce son premier discours à la Chambre lors de l'ouverture de la séance de mars 1643 (Arch. dép. de Loire-Atlantique, 103J 18). 


\section{À l'arrière plan du conflit}

De l'affaire, il nous reste le rapport expédié au roi par le magistrat, les traces documentaires de ses suites et la réminiscence d'événements annexes riches de leçons sur l'existence fonctionnelle des robins. À la suite de la plainte premier président, le Conseil d'Etat promulgue, le 13 décembre 1642, un arrêt en sa faveur, confirmé par lettres patentes du même jour et enregistré à la Chambre le 21 janvier $1643^{14}$. Les préséances y sont rappelées fermement et les contrevenants sommés de s'y conformer :

" [...] Le Roy en Son Conseil a ordonné et ordonne que lesdits arrests seront executtez et ce faisant que ledit Blanchard en laditte quallité de premier president poura touttes fois et quantes bon luy semblera entrer aux deux semestres de ladite Chambre, présider faire les ouvertures et toutes autres fonctions de sadite charge, faict defense ausdits sieurs présidents du Pont, Le Feron et Juchault de les troublés apaine de suspence de leurs charges [...]."

La légitimité de son attitude est renforcée en vertu de sa position de représentant de l'autorité monarchique qu'il relaye dans la province. CésarAuffray Blanchard appuie sa conduite sur la délégation de puissance obtenue par une transaction scellée autour du berceau de l'État naissant où se rencontrent l'ambition des forces sociales dominantes et la volonté de modernisation d'une monarchie administrative ${ }^{15}$. En technicien de la justice responsable, le magistrat s'attache à la définition même de son pouvoir rappelée dans les ouvrages fondamentaux qui peuplent les bibliothèques des gens de justice :

"La puissance des Officiers vient du Prince souverain, auquel Dieu, lors qu'il l'a estably, a remis \& confié la puissance temporelle de son Royaume ${ }^{16}$. "

Mais le magistrat est plus qu'un simple exécutant des ordres royaux, il est le gardien du respect des lois inspirées par le souffle divin. À ce titre sa personne est revêtue d'une forme de sacralité " et partant c'est une des espèces de crime de leze-majesté d'attanter à la personne d'un Officier, quel qu'il soit, estant en l'acte de son Office car ils sont en la sauve-garde spéciale du Prince souverain duquel ils sont la plus haute fonction ${ }^{17}$ ". La réaction du roi apparaît donc comme attendue de la part du premier président, et même de ses contradicteurs, auxquels on enseigne qu'ils forment les fondements de la monarchie.

Toutefois, cette querelle constitue la partie émergée d'un malaise plus profond qui empoisonne les rapports des deux adversaires. Le 22 juin 1644,

14. Idem, B 78.

15. Voir Robert DESCIMON, " Les élites du pouvoir et le prince : l'État comme entreprise " dans : Wolfgang KAISER (dir.), Les élites du pouvoir et la construction de l'État en Europe, Paris, 1996, p. 133-162.

16. Charles Loyseau, Cinq livres du droit des offices, Paris, chez Abel l'Angelier, 1610, chapitre VI, Du pouvoir des officiers.

17. Bernard de La Roche-Flavin, Treize livres des Parlemens de France, Bordeaux, chez Simon Millanges, 1617, livre X, Privilèges, immunités \& exemptions des Présidens, Conseillers \& autres officiers des Parlemens. 
le président René Le Ferron et le maître Jean Fourché ${ }^{18}$, demandent à la reine régente, au nom de leurs collègues, de renouveler les "lettres d'honneur ", données le 23 juin 1643, en faveur de Louis du Pont qui lui permettent d'avoir entrée et voix délibérative avec privilèges et franchises aux audiences " fort et excepté des gaiges ", en vertu des notables services qu'il a rendus sous les règnes des deux souverains précédents ${ }^{19}$. Avant de formuler leur requête, ils s'enquièrent auprès du magistrat qui a " esté requis adverty par le greffier dentrer au Bureau pour le service du Roy ". Une fois installé, on lui demande "si le bruit courran estoit veritable quil sen feust demis et sil estoit daccord contant et satisfait qung autre sy presentat ". Touché par " tant de graces", le président s'excuse de ne pouvoir " respondre assez dignement ". Mais, la cour ne peut vérifier et enregistrer l'acte que le 12 mai 1645 ; celui-ci fut confirmé en mars par lettres de surannation ${ }^{20}$. Le responsable du retard pris par cette validation est le premier président qui s'y est jusqu'à présent opposé malgré le concours d'une partie de la compagnie. Mais cette fois, César-Auffray Blanchard, qui s'incline devant la persévérance des magistrats appuyés par la volonté royale, " a cy devant recogneu ne debvoir cognoistre de ses affaires [...] se retireroit et abstiendroit d'y prendre cognoissance comme plus au long ladite requete le contient ". Le corps déclare accepter l'abstention de son chef " ayant esgard a ce qui sest passé a sa cognoissance " entre les deux individus. Plus encore, la mésentente des deux magistrats semble dater de 1641 lorsqu'une première demande formulée, en mars, par les officiers était restée sans suites faute de soutien. Enfin, on peut imaginer que le premier président a abordé cette question lors de la visite qu'il a faite au Conseil de sa majesté en mars $1643^{21}$.

En dépit de la parenté qui lie les deux personnages, la pression des rangs s'exerce sur eux; outre la rivalité simplement de l'étiquette, le désaccord naît également de la différence d'âge et en amont de l'inégale expérience. Par sa jeunesse, César-Auffray Blanchard dispose encore d'un crédit relatif et contestable aux yeux d'officiers rompus aux arguties de la justice financière; avec la réputation lignagère, le nombre des années de service confère le respect ${ }^{22}$.

D'autre part, en faisant des recherches dans les archives de l'institution, on apprend qu'un incident semblable, dans son enjeu, s'est produit une

18. Jean Fourché (1582-1646) est reçu le 26 janvier 1621 sur résignation de son père Jean. En 1645, il cède son office à François Guischard (1609-1672).

19. Arch. dép. de Loire-Atlantique, B 276.

20. Ibidem.

21. Idem, B 270, arrêt du 18 mars 1643.

22. Bibliothèque Mun. de Nantes, manuscrit 1288, Inventaire du livre blanc de la Chambre des comptes (anonyme, XVII ${ }^{\mathrm{e}}$ siècle), Table du cérémonial commencé le premier mars 1622, "Deces de m[onsieu]r le president de la musse le $6^{\text {eme }}$ apvril 1671 ": "Visite à la veufve et à ses enfants après son decès en laquelle on luy tesmoigna la part que prenoit la compagnie en leur affliction et en la perte quils auront faict d'une personne si considérable pour ses rares vertus que elle ne manqueroit de rendre en touttes occasions les honneurs deubs à sa mémoire et de faire connoistre avec combien de ressentiment elle conservera le souvenir des bons services quelle en a receu. " 
décennie auparavant. En effet, on rapporte que le mercredi premier septembre 1632, le premier président Louis de Harouys ${ }^{23}$ fut " outragé " par un de ses éminents collègues, le président... Louis du Pont. Après s'être fait présenter les clés par les maîtres Mathurin Boux ${ }^{24}$, Jacques Ménardeau ${ }^{25}$ et le premier huissier Hervé Bertaud, il procède à l'ouverture des portes puis, conduit le corps rassemblé à l'église des Cordeliers pour y entendre la messe du Saint-Esprit "à la manière acoustumée ». À leur retour, les magistrats du semestre assistent au coup d'éclat du second président qui exprime son ardent désir de présider le début de la session; ce fut déjà considéré comme "prejudiciable a l'ordre de la justice ${ }^{26}$ ". Déjà, en 1624, le président du Pont, fidèle à sa réputation d'homme colérique, avait perdu son sang froid en portant la main sur le premier président Jacques Barrin qui prétendait pouvoir présider aux deux semestres et céder sa place " selon son bon plaisir ${ }^{27}$ ". Au nombre des mobiles de l'intriguant président, on peut ajouter celui de la convoitise du poste suprême de la Chambre.

Finalement, la péripétie de 1642 est oubliée et disparaît du champ clos où elle a vu le jour. Dans les années suivantes, le premier président n'ignore pas les faveurs royales. Ainsi, le 27 avril 1643; il est accueilli par le chancelier Pierre Séguier au Conseil d'État et privé28. En récompense de ses loyaux services, notamment en la Chambre, et " désirant luy donner moyen de sy entretenir avecq l'honneur et splendeur convenable a la dignité " de son emploi, le roi lui octroie, le 8 novembre 1644, une augmentation de sa pension de 1500 livres $^{29}$.

Par son ascendant institutionnel et la largeur de ses attributions, le poste de premier président s'avère fort exposé comme le remémorent les mésaventures subies par les différents titulaires dans le passé et l'avenir ${ }^{30}$. L'exposé plaintif du premier président, même s'il est empli de subjectivité, nous offre un nouvel épisode de la bataille perpétuelle des préséances qui agite les cours du royaume aux temps modernes. De la confrontation des

23. Le 8 août 1626, Louis de Harouys est le successeur de Jacques Barrin (1578-1645); il délaisse sa charge en 1632 à François Becdelièvre (1599-1633), gendre et prédécesseur de Jean Blanchard (1575-1650) et beau-frère de César-Auffray Blanchard.

24. Mathurin Boux (1594-1670) entre en la Chambre le 5 février 1626 au poste de Pierre Kerboudel ( $\uparrow 1605)$ puis y renonce en 1650 en faveur de Louis du Pas (1621-1673).

25. Jacques Ménardeau († 1633) succède à son père Pierre († 1633) ; il est reçu le 20 janvier 1623. En 1634, la charge est vendue à Alexandre de Complude († 1605).

26. Arch. dép. de Loire-Atlantique, 103J 17, relation anonyme des faits.

27. Henri de Fourmont, op. cit., p. 169. Jacques Barrin (1578-1645) prend la place de Auffray de Lescouet (1561-1632), parrain de César-Auffray Blanchard; il entre en charge le 22 juin 1616 et résigne en faveur de Louis de Harouys en 1626.

28. Arch. dép. de Loire-Atlantique, 103J 17, lettres données le 3 janvier 1642. Le président Tubeuf, surintendant des finances, domaines et affaires d'Anne d'Autriche l'accueille le 6 décembre 1654 au Conseil de la reine régente.

29. Idem, B 78, lettres enregistrées le 12 mai 1645. Cette somme s'ajoute aux 2000 livres qui lui sont allouées depuis le 14 décembre 1641 et que percevait déjà son père et prédécesseur (Arch. dép. de Loire-Atlantique, B 77)

30. Voir annexe. 
codes en vigueur et des aspirations mentales des individus, naît la mise en mouvement du mécanisme des luttes d'influence au sein de la Chambre des comptes de Bretagne révélant la part de clientélisme dans les rapports internes, notamment entre auxiliaires de justice et magistrats supérieurs. Quant au magistrat " chahuté ", il ne manque pas de brandir, pour la sauvegarde de ses prérogatives, le topo du juge-intermédiaire entre le roi et ses sujets qui émaille la rhétorique juridique et croît à l'ombre de l'absolutisme adolescent ${ }^{31}$.

\section{Annexe - « Lettre du Roy touschant la manière dont on doit appeler le s[ieu]r premier president » (Arch. dép. de Loire-Atlantique, B 144, 1686)}

"De par le Roy"

"Nos amez et feaux. Nous avons esté pleinement informez de ce qui s'est passé le $20^{\mathrm{e}}$ febrier dernier, entre le sieur de la Busnelaye ${ }^{32}$, premier president, et autres officiers de n[ot]re Chambre des Comptes de Nantes, et ainsy que vous avez contrevenu à l'usage cy devant observé en lad[ite] Chambre en ne rendant point aud[it] $\mathrm{s}$ [ieu]r de la Busnelaye les honneurs qui luy sont deus en qualité de chef de v[ot]re compagnie, et voulant prevenir pour lavenir de pareilles contesta[ti]ons comme contraires au bien de $\mathrm{n}$ [ot]re service, nous vous escrivons cette lettre pour vous dire que notre intention est que lors que led[it] s[ieu]r premier president se retirera du bureau pou y laisser opiner, il soit convié par un m[aîtr]e des comptes, apres les delibéra[ti]ons prises, dy rentrer pour reprendre sa place, et aussy pour vous faire sçavoir que nous desirons que v[ot]re compagnie depute deux m[aître]s d'entre elle pour aller tesmoigner de sa part aud[it] sieur de la Busnelaye le desplaisir qu'a vostre d[ite] compagnie de ce qui sest passé, et lassure quelle se conformera à lavenir à ce qui est en cela de nostre volonté. Ny faictes donc faute. Car tel est notre plaisir, donné à Versailles le premier jour de juin 1686. - Signé : Louis "

\section{RESUME}

En septembre 1642, l'ouverture de la séance de la Chambre des comptes de Bretagne est troublée par des officiers qui contestent l'autorité du premier président César-Auffray Blanchard. Cette querelle qui n'est ni la première, ni la dernière, permet d'étudier, par l'énoncé des faits et leur interprétation, le comportement des hauts magistrats de province au $\mathrm{XVII}^{\mathrm{e}}$ siècle.

\section{ABSTRACT}

In september 1642, opening ceremony of Chamber of counts of Brittany was perturbed by officers who contest authority of prime president. This disput, that's neither the first nor the last, allow to study behaviours of provincial judges through events and interpretation in XVII th Century.

31. Francesco di DonATo, "Constitutionnalisme et idéologie de robe. L'évolution de la théorie juridico-politique de Murard et Le Paige à Chanlaire et Mably ", Annales, Histoire, Sciences Sociales, $\mathrm{n}^{\circ}$ 4, 1997, p. 821-838.

32. Jean-Baptiste Becdelièvre de La Busnelaye (1651-1736), est reçu le 5 septembre 1678 au poste de Jacques Charette de Montbert (1637-1677) et se retire en 1722 en faveur de son fils Guillaume. Il est le fils de Jean-Baptiste Becdelièvre (1625-1656), avocat général à la Chambre puis, président au Parlement de Bretagne, petit-fils du premier président François Becdelièvre (1599-1633) et petit-neveu de César-Auffray Blanchard. 